\title{
Comparison of Virulence between Five Strains of Cryptococcus Species Complex in a Rat Model
}

\author{
Gyu-Nam Park ${ }^{1, *}$, Sun-Young Kim ${ }^{2, * *}$, Hye-Ran Kim ${ }^{1, * *}$, Bo-Kyung Jung ${ }^{1, *}$, \\ Dong-Jun $\mathrm{An}^{3, * *}$, Seung-Bok Hong ${ }^{4, * * *}$ and Kyung-Soo Chang ${ }^{1, \dagger ; * * *}$ \\ ${ }^{I}$ Department of Clinical Laboratory Science, College of Health Sciences, \\ Catholic University of Pusan, Busan 46252, Korea \\ ${ }^{2}$ Department of Pathology, Dongnam Institute of Radiological and Medical Sciences, Busan 46033, Korea \\ ${ }^{3}$ Animal and Plant Quarantine and Inspection Agency (QIA), Gyeongsangbuk-do 39660, Korea \\ ${ }^{4}$ Department of Clinical Laboratory Science, Chungbuk Health and Science University, \\ Chungbuk 28150, Korea
}

\begin{abstract}
Cryptococcosis, which is caused by the Cryptococcus species complex (including Cryptococcus neoformans and Cryptococcus gattii), is well known as one of the most important medical problems. However, the of the Cryptococcus species complex is still limited to pneumonia and meningitis. In particular, the differences in virulence among the five major serotypes of the Cryptococcus species complex are not fully understood. To elucidate the virulence of the Cryptococcus species complex when it is disseminated hematogenously, rats were infected by different strains of the Cryptococcus species serotype, and their histopathological characteristics were compared after infection. The cumulative mortality ratio of rats infected with serotype B strain was slightly higher than in the other experimental groups. In addition, the average recovery of the Cryptococcus species complex from rats infected with serotype B strain was significantly higher than in the other groups in almost all organ samples except spleen. The recovery of the Cryptococcus species complex was associated with the severity of histopathological lesions, including bleeding, inflammation, and tissue damage in all organs. In rats infected with serotype B strain, the virulence was the most severe, especially in the lungs and liver. These results indicate that the pathophysiology of the Cryptococcus species complex infection differs according to serotype.
\end{abstract}

Key Words: Cryptococcus species complex, Serotype, Virulence, Histopathological change, Rat model

\section{INTRODUCTION}

The Cryptococcus species complex (including Cryptococcus neoformans and Cryptococcus gattii) is an opportunistic fungal pathogen that causes the life-threatening infection, Cryptococcosis. This pathogen is distributed world- wide, and major habitats are avian droppings and contaminated soil (Buchanan and Murphy, 1998; Hansong and Robin, 2009; Negroni, 2012). The infection is initiated primarily by the inhalation of cryptococcal infectious particles emitted from environmental materials, which can cause infection in the lung and central nervous system (CNS) in human and animal hosts, particularly in immunocompromised patients.

Received: June 14, 2018 / Accepted: August 23, 2018

*Graduate student, ${ }^{* *}$ Researcher, ${ }^{* * *}$ Professor.

${ }^{\dagger}$ Corresponding author: Kyung-Soo Chang. Department of Clinical Laboratory Science, College of Health Sciences, Catholic University of Pusan, Busan 46252, Korea.

Tel: +82-51-510-0565, Fax: +82-51-510-0568, e-mail: kschang@cup.ac.kr

(C) The Korean Society for Biomedical Laboratory Sciences. All rights reserved.

(C) This is an Open Access article distributed under the terms of the Creative Commons Attribution Non-Commercial License (http://creativecommons.org/licenses/by-nc/3.0/) which permits unrestricted non-commercial use, distribution, and reproduction in any medium, provided the original work is properly cited. 
It is generally known that the major infection sites of the Cryptococcus species complex are brain and lung. These fungal pathogens may spread hematogenously to other organ systems (Buchanan and Murphy, 1998; Lortholary et al., 1999; Hansong and Robin, 2009; Negroni, 2012).

Cryptococcosis has been responsible for high morbidity and mortality rates, particularly in AIDS patients. Globally, approximately 1 million cases of meningitis occur each year in AIDS patients, resulting in approximately 624,700 deaths within three months after infection by the Cryptococcus species complex (Centers for Disease Control and Prevention, CDC, Atlanta, USA, http://www.cdc.gov/). In addition, the incidence of cryptococcosis has increased in recent years because of the large increase in the number of AIDS patients, population aging, and the expanded use of immunosuppressive drugs for cancer treatment, organ transplantation, etc. (Park et al., 2009; Michael and Christina, 2010; Cogliati, 2012). Therefore, cryptococcosis is regarded as one of the most important opportunistic fungal diseases.

The Cryptococcus species complex is currently classified into two species and five major serotypes. Cryptococcus neoformans (C. neoformans) and Cryptococcus gattii (C. gattii). Because of the differences in ecological, biochemical, and molecular features, C. gattii (including serotypes B and C) has been defined as a separate species. C. neoformans is divided into two varieties: $C$. neoformans var. grubii (including serotypes A) and $C$. neoformans var. neoformans (including serotypes D and A/D) (Chen et al., 2011). The differences between Cryptococcus species complex are associated with epidemiology, pathogenicity, and geographic distribution. C. neoformans var. grubii (serotype A) is predominant strain of $C$. neoformans that is isolated from infected patients and is distributed worldwide and responsible for more than $90 \%$ of the cases of systemic Cryptococcosis. $C$. neoformans var. neoformans (serotypes D and A/D) occurs worldwide but mainly in temperate climates, such as Europe and South America. In comparison, the geographical distribution of C. gattii (serotypes B and C) is more restricted than C. neoformans, which is in tropical and subtropical regions (Chen et al., 2011; Negroni, 2012). However, recent studies have reported its worldwide distribution, such as in British Columbia (Canada) and the Pacific Northwest in the US
(Nielsen et al., 2007; Chaturvedi and Chaturvedi, 2011; Ngamskulrungroj et al., 2011). Although cases of Crytococcosis caused by $C$. gattii are rare, compared with $C$. neoformans, they can cause life-threatening infections in immunocompetent hosts (Negroni, 2012).

Cryptococcosis that is caused by the Cryptococcus species complex is well known as one of the most important medical problems worldwide. However, cryptococcosis that is caused by the different cryptococcal serotypes is not fully understood. In this study, we compared the histopathological changes of rats intravenously infected by different serotypes of cryptococcal strains.

\section{MATERIALS AND METHODS}

\section{Animals}

Six-week-old male Sprague Dawley (SD) rats (weighing 110 150 grams) were purchased from Samtako Bio Korea, Inc. (Osan, Korea). The rats were raised under specificpathogen-free conditions in cages covered by a filter top and were given clean food and water ad libitum. All rats were adapted to a 12-hour light/dark cycle at a temperature of $25^{\circ} \mathrm{C}$ for one week. The Animal Care and Use Committee of the Catholic University of Pusan (CUP-AEC 2012-02) approved this study.

\section{Fungal strains}

C. neoformans serotype D (ATCC 66031), serotype A/D (ATCC 48184) strains, and C. gattii serotype B (MYA-4560), serotype C (ATCC 32608) strains were used in this study. These Cryptococcus species complex strains were purchased from the American Type Culture Collection (ATCC). One clinical strain (CS), identified as C. neoformans var. grubii, which was provided by the general hospital in Busan, Korea. This strain was used as the serotype A of C. neoformans. All cryptococcal strains were stored at $-70^{\circ} \mathrm{C}$ in a freezer until they were used.

\section{Cultures of Cryptococcus species complex}

To prepare the working fungal solution, all $C$. neoformans and $C$. gattii strains were recovered from frozen glycerol stocks. One hundred micro-liters from glycerol stock was 
Table 1. Experimental design of animal study for Cryptococcus species complex by serotype

\begin{tabular}{ccll}
\hline \hline Groups & Animal $(\mathrm{n})$ & \multicolumn{1}{c}{ Inoculums $\left(2.0 \times 10^{6}\right.$ cells $\left./ 100 \mu \mathrm{L}\right)$} & \multicolumn{1}{c}{ Injection } \\
\hline I & 5 & C. neoformans var. grubii clinical strain & 1,3 , and 5 weeks \\
II & 5 & C. gattii (ATCC MYA-4560) & 1,3 , and 5 weeks \\
III & 5 & C. gattii (ATCC 32608) & 1,3 , and 5 weeks \\
IV & 5 & C. neoformans var. neoformans (ATCC 66031) & 1,3 , and 5 weeks \\
V & 5 & C. neoformans var. neoformans (ATCC 48184) & 1,3 , and 5 weeks \\
Control & 2 & Sterile saline & 1,3 , and 5 weeks \\
\hline Total & 27 & &
\end{tabular}

inoculated onto a sunflower seed (Helianthus annus) agar (SSA, the selective culture medium for Cryptococcus species complex) plate containing $0.1 \%$ creatinine and $40 \mathrm{~mL}$ chloramphenicol $(50 \mu \mathrm{g} / \mathrm{mL})$ to detect the typical brown pigmentation of the Cryptococcus species complex. The plates were incubated in the dark at $37^{\circ} \mathrm{C}$ under a humid condition. After five days, brown colonies of each strain were recovered and then transferred into $10 \mathrm{~mL}$ Sabouraud dextrose broth (SDB) before they were incubated for 48 hours at $37^{\circ} \mathrm{C}$ in a shaker. Each culture suspension was centrifuged at 6,500 $\times \mathrm{g}$ for $1 \mathrm{~min}$; the supernatant was then discarded. The fungal pellets were washed three times with sterilized phosphate buffered saline (PBS), and the fungal cells were counted with a hemocytometer. The cryptococcal cells of each strain were diluted to obtain a concentration of $2.0 \times 10^{7}$ cells/ $\mathrm{mL}$ in sterilized PBS.

\section{Design of animal study}

After adaptation for one week, the rats were divided into 6 groups ( 5 heads in each group, except for negative control) and treated as shown in Table 1. Briefly, experimental groups I to $\mathrm{V}(1,3$, and 5 weeks) were infected by the injection of $100 \mu \mathrm{L}$ (approximately $2.0 \times 10^{6}$ cells/rat) inoculums of the Cryptococcus species complex serotype A, B, C, D and $\mathrm{AD}$, respectively. The control group received with sterile saline. All groups were injected intravenously (tail vein injection) using a 26 gauge needle attached to a $1 \mathrm{~mL}$ syringe. All live rats in the experimental and control groups were sacrificed at six weeks after the first infection.

\section{Histopathological findings}

All experimental rats were anesthetized through the inhalation of ethyl ether. For the histopathological examination, the lung, brain, heart, liver, spleen, kidney, testis, and muscle tissues were aseptically collected from the sacrificed rats. The collected organs were fixed in $10 \%$ neutral buffered formalin for 24 hours at room temperature. A portion of each organ (left hemisphere of lung, kidney, and brain and segments of liver and spleen tissues) was separately collected for recovery of the infected Cryptococcus species complex. The organs were fixed in paraffin-embedded histological cassettes. The paraffin-embedded organ blocks were cut into $5-\mu \mathrm{m}$ sections and then were stained with periodic acidSchiff (PAS). To detect histopathological characteristics, such as inflammation, bleeding, tissue damage, and cryptococcal cells, all tissue sections were observed by light microscopic examination.

\section{Culture for recovery of Cryptococcus species complex from the various organs}

For recovery of the Cryptococcus species complex from the various organs of the infected rats, a portion of each organ was homogenized in $10 \mathrm{~mL}$ of PBS; $100 \mu \mathrm{L}$ of the organ homogenates were plated onto SSA plates and incubated for $48 \sim 72 \mathrm{hr}$ at $37^{\circ} \mathrm{C}$. Blood for culturing was collected directly from the heart of the infected rats. One milliliter of each blood sample was inoculated onto the SSA plates and incubated at $37^{\circ} \mathrm{C}$ under a humid condition. After incubation, the brown pigmented colonies were counted to calculate the recovery rate of the Cryptococcus species com- 
plex. The number of the brown pigmented colonies was normalized according to organ weight.

\section{Identification of Cryptococcus species complex}

Genotyping was used to identify the recovered single pure colonies. The genotyping of the fungal pathogens was performed as previously described (Chae et al., 2012). Briefly, each pure colony from the organ samples was cultured on SDB for 48 hours at $25^{\circ} \mathrm{C}$. One milliliter of cultivated broth was moved to a new tube and centrifuged. The sample pellet was suspended in $500 \mu \mathrm{L}$ of extraction buffer $(100 \mathrm{mM}$ Tris $\mathrm{HCl}$ [pH 9.0], $40 \mathrm{mM}$ EDTA) and then mixed after the addition of $100 \mu \mathrm{L}$ of $10 \%$ sodium dodecyl sulfate (SDS) and $300 \mu \mathrm{L}$ of $99 \%$ benzyl chloride. The mixture was homogenized and incubated at $50^{\circ} \mathrm{C}$ for 30 min with gentle shaking. After centrifugation, the supernatant was transferred to a new tube. After the addition of $1 / 10$ volume of $3 \mathrm{M}$ sodium acetate and an equal volume of isopropyl alcohol in the supernatant, the tube was slightly vortexed and then stored at $-70^{\circ} \mathrm{C}$ for $1 \mathrm{hr}$. The DNA was precipitated after centrifugation, and the supernatant was removed. The pellets were washed with $500 \mu \mathrm{L}$ of ethanol, and then the supernatant was removed by centrifugation. The pellet was dried at room temperature for $10 \mathrm{~min}$ and then resuspended in $50 \mu \mathrm{L}$ of distilled water (D.W.). The extracted genomic DNA was stored at $-70^{\circ} \mathrm{C}$ until use.

PCR was performed using the AccuPower PCR premix Kit (Bioneer, Seoul, Korea). A total volume of $20 \mu \mathrm{L}$ of the reaction mixture was contained within each $1 \mu \mathrm{L}$ of primer $(10 \mathrm{pM} / \mu \mathrm{L}), 2 \mu \mathrm{L}$ of template DNA $(1 \mu \mathrm{g} / \mu \mathrm{L})$, and $16 \mu \mathrm{L}$ of D.W. The amplification of the $C N L A C l$ gene was carried out using the following conditions: initial denaturation at $95^{\circ} \mathrm{C}$ for $5 \mathrm{~min} ; 35$ cycles of $95^{\circ} \mathrm{C}$ for $1 \mathrm{~min} ; 58^{\circ} \mathrm{C}$ for 1 min; $72^{\circ} \mathrm{C}$ for $90 \mathrm{sec}$; and a final extension at $72^{\circ} \mathrm{C}$ for 7 $\mathrm{min}$. The PCR products were analyzed by electrophoresis with $1.2 \%$ agarose gels containing ethidium bromide and were visualized under UV light. The amplified DNA fragments in the gel were purified using the Accuprep ${ }^{\circledR}$ Gel Purification Kit (Bioneer, Seoul, Korea) and identified by DNA sequence analysis.

\section{Statistical analysis}

All experimental data were expressed as mean \pm standard deviation (S.D.). The mortality of rats infected with the fungal pathogen was analyzed using the Kaplan-Meier survival curve with a log-lank significance test. The cryptococcal colonies recovered from homogenates were analyzed using a one-way analysis of variance (ANOVA) with the Scheffe post hoc test. All statistical analyses were performed using the programs SPSS version 20.0 and Microsoft Excel. A $P$-value $<0.05$ was considered statistically significant.

\section{RESULTS}

\section{Mortality of rats infected with Cryptococcus species com- plex}

The cumulative mortality of rats infected with Cryptococcus species complex differed according to serotype (Fig. 1). After the first infection with the Cryptococcus species complex, no rat mortality was observed for about 18 days. In experimental group II, rat mortality occurred primarily on the 19th day after the first infection. Rat mortality occurred in groups II and III on the 26th and 32nd days after the second infection, respectively. Only one rat infected with $C$. neoformans strains died on the 23rd day post-infection (pi).

In group II, which was infected with $C$. gattii MYA-4560 (serotype B), two rats died $(2 / 5,40.0 \%)$ during the experiment. During the experiment, one rat died in group III, which was infected with C. gattii 32608 (serotype C). One rat died in group IV, which was infected with C. neoformans 66031 (serotype D) $(1 / 5,20.0 \%)$. No mortality occurred in group I, which was infected with C. neoformans var. grubii (serotype A) or in group V, which was infected with C. neoformans 48184 (serotype A/D) (0/5, 0.0\%).

To determine the differences in pathogenicity between $C$. gattii and C. neoformans, the Kaplan-Meier survival analysis with a log-lank significance test was performed by using SPSS version 20.0. The cumulative mortality of rats infected with $C$. gattii $(3 / 10,30.0 \%)$ was higher than that of rats infected with $C$. neoformans (1/15, about 6.7\%). A significant difference was not found $(P=0.130)$ in the cumulative mortality of rats infected with $C$. gattii (serotypes B and C) 
Table 2. The numbers of recovered cryptococcal colonies from organs of the rats infected by Cryptococcus species complex

\begin{tabular}{cccccc}
\hline \hline \multirow{2}{*}{ Groups } & \multicolumn{5}{c}{ Organs } \\
\cline { 2 - 6 } & Lung & Liver & Kidney & Brain & Spleen \\
\hline I & $92.80 \pm 17.60$ & $34.60 \pm 7.47$ & $31.40 \pm 9.74^{*}$ & $43.00 \pm 11.24$ & $14.80 \pm 4.32$ \\
II & $182.33 \pm 22.37^{*}$ & $155.00 \pm 8.89^{*}$ & $94.33 \pm 10.21$ & $92.67 \pm 8.50^{*}$ & $84.67 \pm 12.66$ \\
III & $95.50 \pm 18.08$ & $46.50 \pm 12.50$ & $65.50 \pm 19.84$ & $51.50 \pm 6.99$ & $99.25 \pm 15.59$ \\
IV & $101.75 \pm 18.00$ & $49.75 \pm 12.91$ & $65.50 \pm 19.84$ & $86.25 \pm 5.44^{*}$ & $93.00 \pm 10.42$ \\
V & $105.00 \pm 13.66$ & $50.80 \pm 12.13$ & $85.00 \pm 7.65$ & $60.60 \pm 14.47$ & $66.00 \pm 5.83$ \\
Control & $0.00 \pm 0.00$ & $0.00 \pm 0.00$ & $0.00 \pm 0.00$ & $0.00 \pm 0.00$ & $0.00 \pm 0.00$ \\
\hline
\end{tabular}

Note. All data are mean \pm S.D.

$*, P<0.05$.

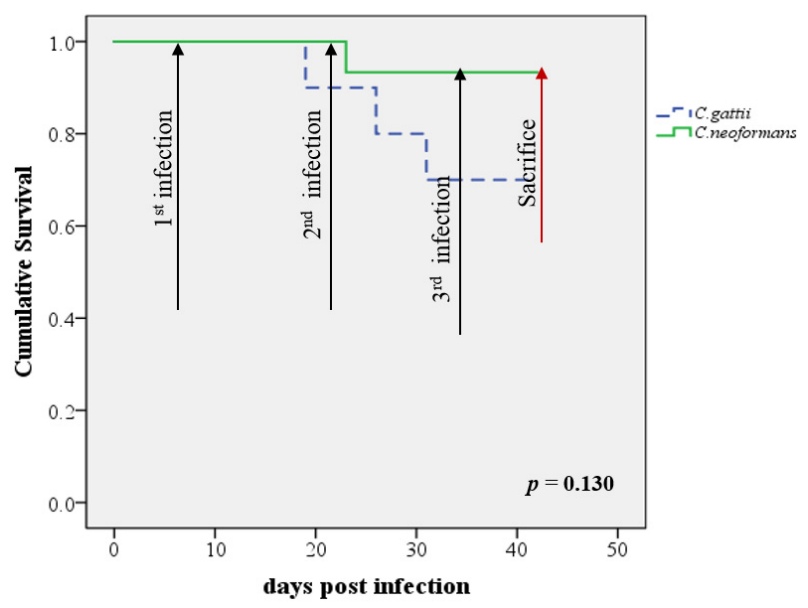

Fig. 1. The survival curves for experimental rats infected with $C$. gattii (MYA-4560 and 32608) and C. neoformans (CS, 66031, and $48184)$ were $3 / 10(30.0 \%), 1 / 15(6.7 \%)$, respectively. Significant difference was not found in mortality between rats infected with $C$. gattii and C. neoformans $(P=0.130)$.

and with C. neoformans (serotypes A, D, and A/D) (Fig. 1).

\section{Recovery of cryptococcal colonies from various organs}

This experiment was performed using organ homogenates from live experimental rats. The recovery of cryptococcal colonies was determined by culturing homogenized organ samples. After incubation for 72 hours on SSA plates, brown pigmented pure colonies were obtained. They were counted in homogenized organ samples of each experimental group except the negative control group. Cryptococcal brown pigmented colonies were recovered from almost all infected organs (lung, liver, kidney, brain, and spleen) but not from the blood samples. Brown cryptococcal colonies (mean \pm S.D) were recovered from all organ culture samples of rats infected with C. gattii MYA-4560 (Group II), 32608 (Group III), C. neoformans 66031 (Group IV), 48184 (Group V), and CS (Group I) in that order (Table 2). Generally, the number of recovered crytococcal colonies was higher in rats infected with C. gattii MYA-4560 than in the other groups. The one-way ANOVA and the Scheffe post hoc test revealed significant differences in the mean number of the recovered colonies in all experimental groups (Fig. 2). In particular, the number of cryptococcal colonies recovered from the lung and liver culture samples was significantly higher $(P$ $<0.05$ ) in rats infected with Cryptococcus species complex serotype B than in the other groups (Fig. 2. (A) and (D)). The number of cryptococcal colonies that were recovered from the kidney and spleen culture samples was significantly lower $(P<0.05)$ in rats infected with Cryptococcus species complex serotype A than in the other groups (Fig. 2. (B) and (E)). The number of cryptococcal colonies that were recovered from the brain culture samples was significantly higher $(P<0.05)$ in rats infected with Cryptococcus species complex serotype B and D than those infected with the other serotypes (Fig. 2. (C)). These results showed that the infection and replication of the Cryptococcus species complex differed according to serotype.

The results of the one-way ANOVA and the Scheffe post hoc test showed that the average number of cryptococcal colonies were recovered from lung samples was significantly higher $(P<0.05)$ than in the other organ samples (Fig. 3). However, cryptococcal colonies were recovered not only 
A

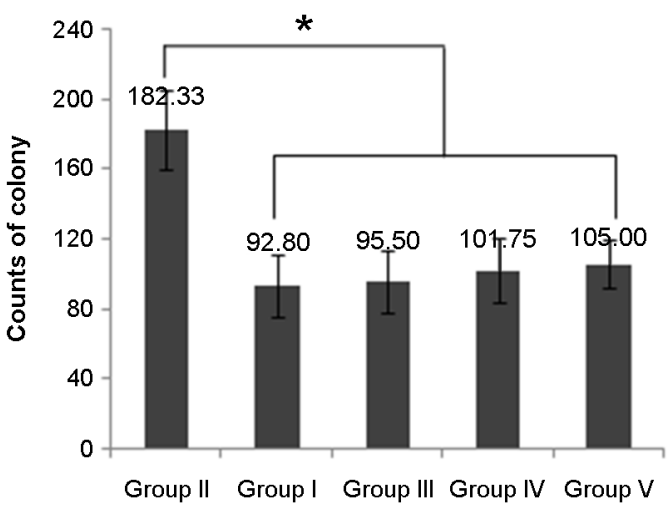

Experimental group

C

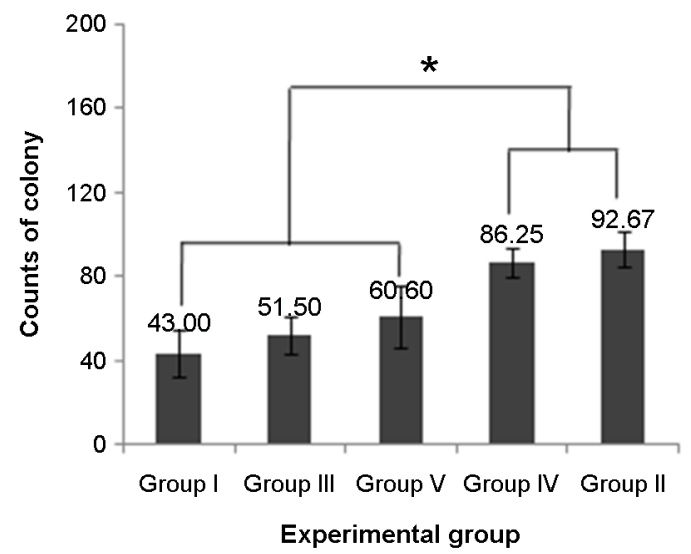

E

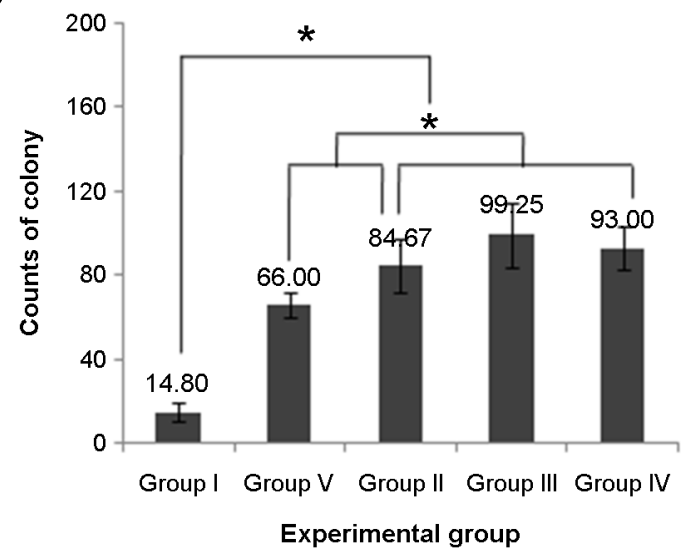

from the lung and brain samples, which are known as major infection sites of Cryptococcus species complex, but also from the kidney, liver, and spleen samples.
B

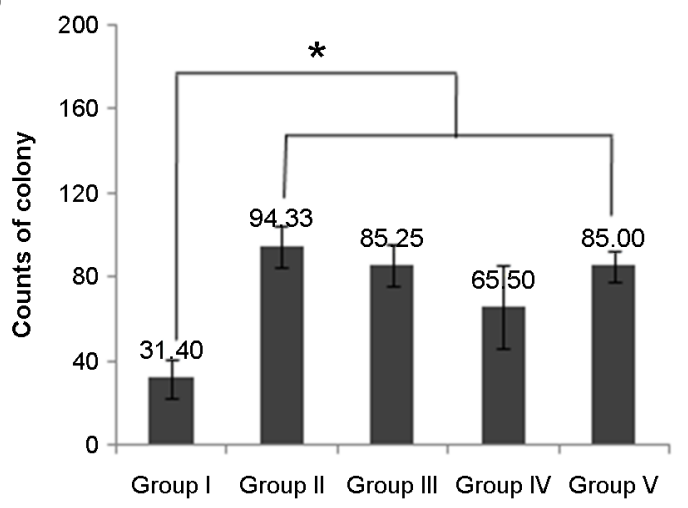

Experimental group

D

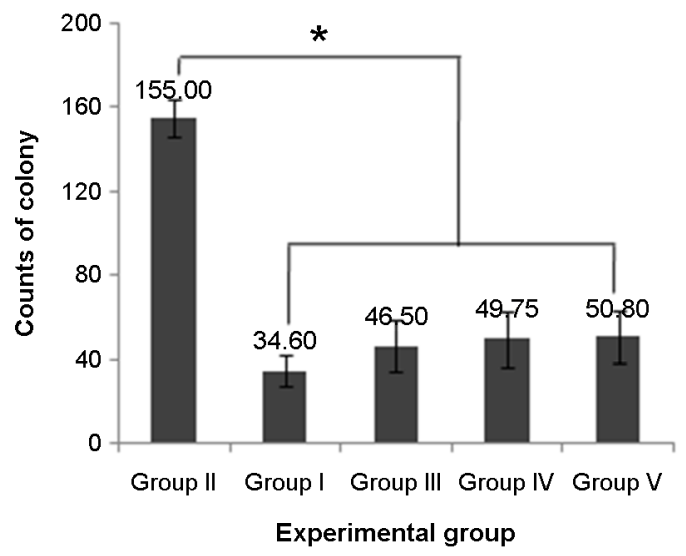

Fig. 2. The results of one-way ANOVA and Scheffe post hoc test for fungal colonies recovered from various organs. (A) The result of one-way ANOVA and Scheffe post hoc test for fungal colonies recovered from lung. (B) The result of one-way ANOVA and Scheffe post hoc test for fungal colonies recovered kidney. (C) The result of one-way ANOVA and Scheffe post hoc test for fungal colonies recovered brain. (D) The result of oneway ANOVA and Scheffe post hoc test for fungal colonies recovered liver. (E) The result of one-way ANOVA and Scheffe post hoc test for fungal colonies recovered spleen. *, $P<0.05$.

\section{Identification of Cryptococcus species complex from organs}

Pure brown pigmented colonies were recovered from the homogenized organ samples in all experimental groups. Every recovered fungal pathogen was identified by using 
the CNLAC1 genotyping method (data not shown). Approximately 1,200 bp of amplified fragment was detected in all recovered fungal pathogens (100.0\%). After the purified DNA was sequenced, the sequences were compared to reference data available in the Genbank database by using a BLAST search to identify the species. The results of the identification by PCR and sequencing were in accordance with the Cryptococcus species complex serotype with which the rats were infected.

\section{Histopathological changes by Cryptococcus species com- plex serotype}

To determine the histological virulence of the rats infected with the Cryptococcus species complex, histological tissue sections (right hemisphere of lung, kidney, and brain and segments of liver, spleen, heart, and muscle tissues) of every experimental group were stained by PAS stain and examined by light microscopy to detect inflammation, bleeding, tissue

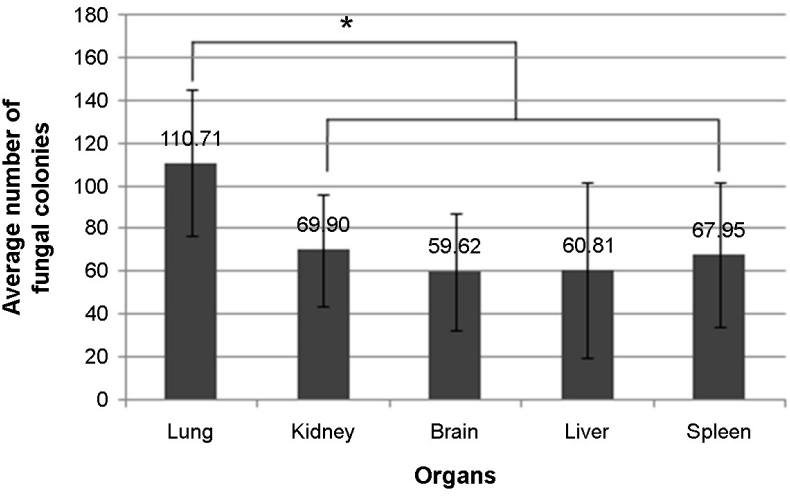

Fig. 3. One-way ANOVA and Scheffe post hoc test revealed significant differences in number of fungal colonies recovered from various organs between lung and the others. ${ }^{*}, P<0.05$.

Table 3. Histopathology degrees for major organ sections from rats infected by Cryptococcus species complex

\begin{tabular}{|c|c|c|c|c|c|c|c|c|c|}
\hline \multirow{3}{*}{ Groups } & \multicolumn{9}{|c|}{ Histopathological degrees of major organ sections } \\
\hline & \multicolumn{3}{|c|}{ Lung } & \multicolumn{3}{|c|}{ Liver } & \multicolumn{3}{|c|}{ Brain } \\
\hline & Bleeding & Inflammation & $\begin{array}{c}\text { Tissue } \\
\text { damage }\end{array}$ & Bleeding & Inflammation & $\begin{array}{c}\text { Tissue } \\
\text { damage }\end{array}$ & Bleeding & Inflammation & $\begin{array}{l}\text { Tissue } \\
\text { damage }\end{array}$ \\
\hline I & $(-)$ & $(+)$ & $(+)$ & $(+)$ & $(-)$ & $(+)$ & $(-)$ & $(+)$ & $(+)$ \\
\hline II & $(+++)$ & $(++)$ & $(+++)$ & $(++)$ & $(++)$ & $(+++)$ & $(++)$ & $(+++)$ & $(++)$ \\
\hline III & $(+)$ & $(++)$ & $(+)$ & $(+)$ & $(+)$ & $(+)$ & $(+)$ & $(++)$ & $(+)$ \\
\hline IV & $(++)$ & $(++)$ & $(++)$ & $(++)$ & $(++)$ & $(++)$ & $(++)$ & $(++)$ & $(++)$ \\
\hline V & $(+)$ & $(++)$ & $(++)$ & $(+)$ & $(++)$ & $(+)$ & $(-)$ & $(+)$ & $(++)$ \\
\hline Control & $(-)$ & $(-)$ & $(-)$ & $(-)$ & $(-)$ & $(-)$ & $(-)$ & $(-)$ & $(-)$ \\
\hline
\end{tabular}

Note. $(-)$; no damage, $(+)$; slight, $(+)$; intermediate, $(+++)$; severe

Table 4. Histopathology degrees of kidney and spleen sections from rats infected by Cryptococcus species complex

\begin{tabular}{|c|c|c|c|c|c|c|}
\hline \multirow{3}{*}{ Groups } & \multicolumn{6}{|c|}{ Histopathological degrees of major organ sections } \\
\hline & \multicolumn{3}{|c|}{ Kidney } & \multicolumn{3}{|c|}{ Spleen } \\
\hline & Bleeding & Inflammation & Tissue damage & Bleeding & Inflammation & Tissue damage \\
\hline I & $(+)$ & $(-)$ & $(+)$ & $(-)$ & $(+)$ & $(-)$ \\
\hline II & $(++)$ & $(++)$ & $(+++)$ & $(++)$ & $(++)$ & $(++)$ \\
\hline III & $(++)$ & $(+)$ & $(++)$ & $(+)$ & $(++)$ & $(++)$ \\
\hline IV & $(+)$ & $(+)$ & $(-)$ & $(+)$ & $(++)$ & $(++)$ \\
\hline V & $(+)$ & $(++)$ & $(++)$ & $(+)$ & $(+)$ & $(+)$ \\
\hline Control & $(-)$ & $(-)$ & $(-)$ & $(-)$ & $(-)$ & $(-)$ \\
\hline
\end{tabular}

Note. $(-)$; no damage, $(+)$; slight, $(++)$; intermediate, $(+++)$; severe 

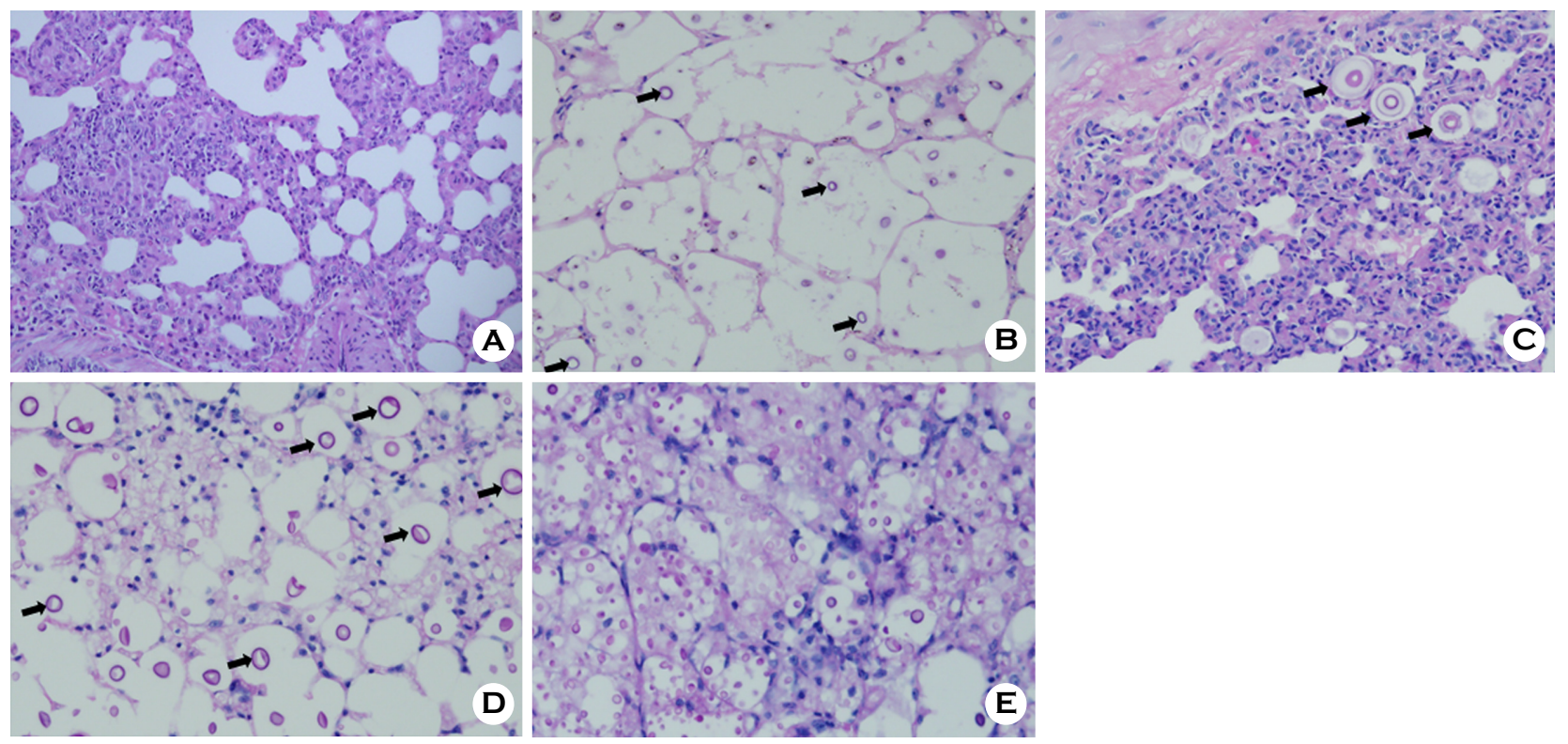

Fig. 4. Lung sections of rats infected with Cryptococcus species complex showed different histopathological characteristics between experimental groups. (A) Histopathological examination of CS (serotype A, group I) infected rat lung sections (Periodic acid Schiff stain, $\times 100$ ). (B) Histopathological examination of C. gattii (serotype B, group II) infected rat lung sections (Periodic acid Schiff stain, $\times 200$ ). (C) Histopathological examination of C. gattii (serotype C, group III) infected rat lung sections (Periodic acid Schiff stain, $\times$ 200). (D) Histopathological examination of $C$. neoformans (serotype D, group IV) infected rat lung sections (Periodic acid Schiff stain, $\times 200$ ). (E) Histopathological examination of C. neoformans (serotype A/D, group V) infected rat lung sections (Periodic acid Schiff stain, $\times 200$ ).

damage, and cryptococcal organism. The results (including inflammation, bleeding, and tissue damage) of the histological examination of rat organs infected with the Cryptococcus species complex showed different degrees of virulence in each experimental group (Table 3 and 4). The most severe bleeding, inflammation, and tissue damage was observed in the tissue sections of group II (especially in lung, liver, and brain sections). In contrast, bleeding, inflammation, and tissue damage were observed slightly in almost all histological sections of group I but were not observed in those of negative control group (data not shown).

Infiltrations by inflammatory and yeast cells in damaged alveoli were observed in the lung sections of the rats in groups IV and V (Fig. 4. (D) and (E)). In group II, the interalveolar septum was severely damaged and infiltrated by yeast cells in the alveoli and alveolar saccules, as shown in Fig. 4. (B). Thickening interalveolar septum around terminal bronchioles, which was caused by inflammation and the infiltration of yeast cells, were observed in group III (Fig. 4.
(C)). Inflammation and tissue damage were observed in group I, but yeat cells were not detected in the lung sections of these rats.

The appearance of macrophages and the infiltration of yeast cells in renal tubules were observed in the kidney sections of group II (Fig. 5. (B)). Inflammation and the infiltration of yeast cells in damaged renal tubules and Bowman's capsule were observed in the kidney sections of groups IV and V (Fig. 5. (D) and (E)). Infiltration of yeast cells in renal tubules was observed in group III (Fig. 5. (C)), but yeast cells were not detected in the kidney sections of group I.

Severe inflammation and infiltration of yeast cells in the germinal center of the lymph node were observed the in spleen sections of group II (Fig. 6. (B)). Increased alveolar macrophage and infiltration of yeast cells in white pulp were observed in group III (Fig. 6. (C)). Infiltration of yeast cells in white pulp was observed in both group IV and group $\mathrm{V}$ (Fig. 6. (D) and (E)), but yeast cells were not detected in the spleen sections of group I. 

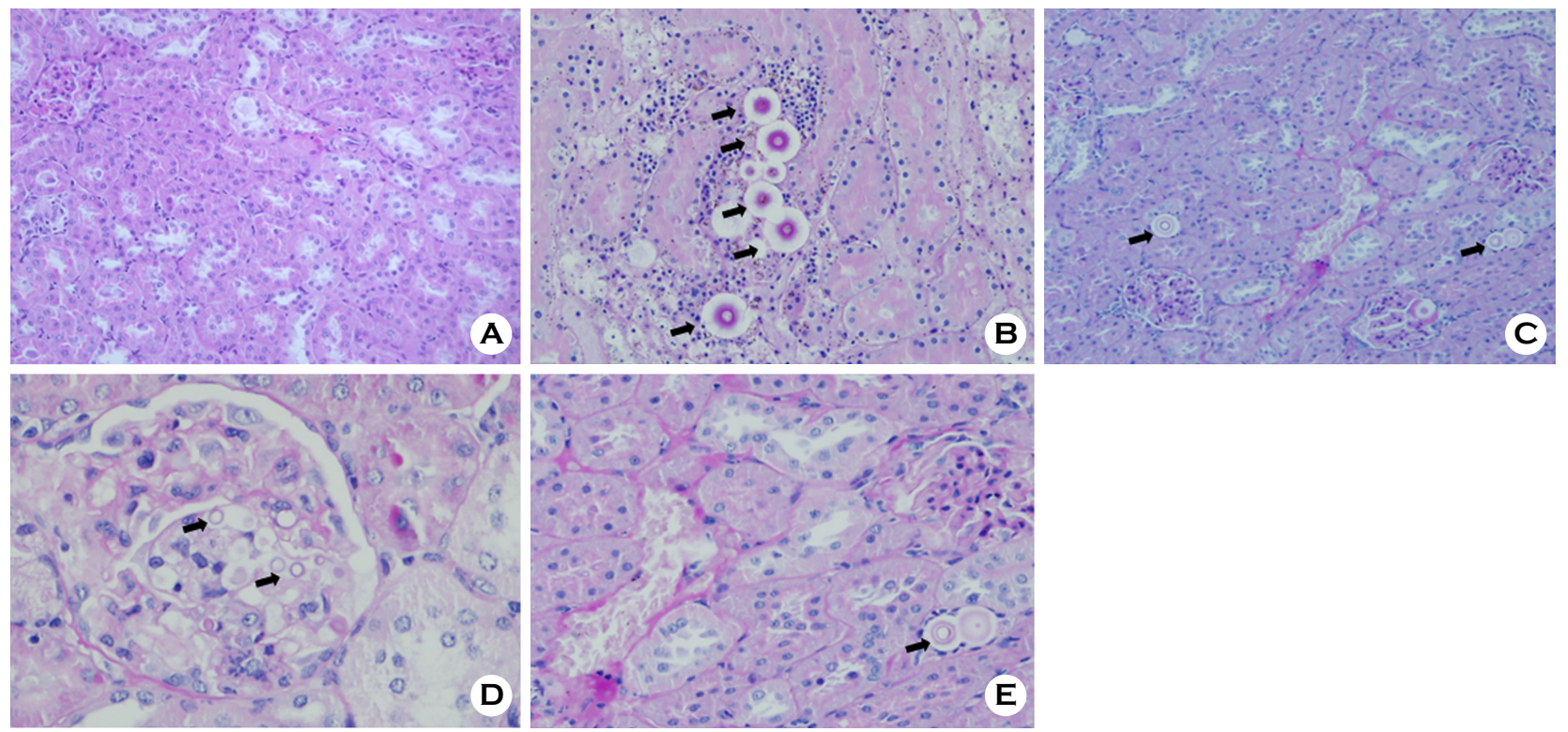

Fig. 5. Kidney sections of rats infected with Cryptococcus species complex showed different histopathological characteristics between experimental groups. (A) Histopathological examination of CS (serotype A, group I) infected rat kidney sections (Periodic acid Schiff stain, $\times$ 200). (B) Histopathological examination of $C$. gattii (serotype B, group II) infected rat kidney sections (Periodic acid Schiff stain, $\times 200$ ). (C) Histopathological examination of $C$. gattii (serotype C, group III) infected rat kidney sections (Periodic acid Schiff stain, $\times 100$ ). (D) Histopathological examination of C. neoformans (serotype D, group IV) infected rat kidney sections (Periodic acid Schiff stain, $\times 200$ ). (E) Histopathological examination of C. neoformans (serotype A/D, group V) infected rat kidney sections (Periodic acid Schiff stain, $\times 200$ ).
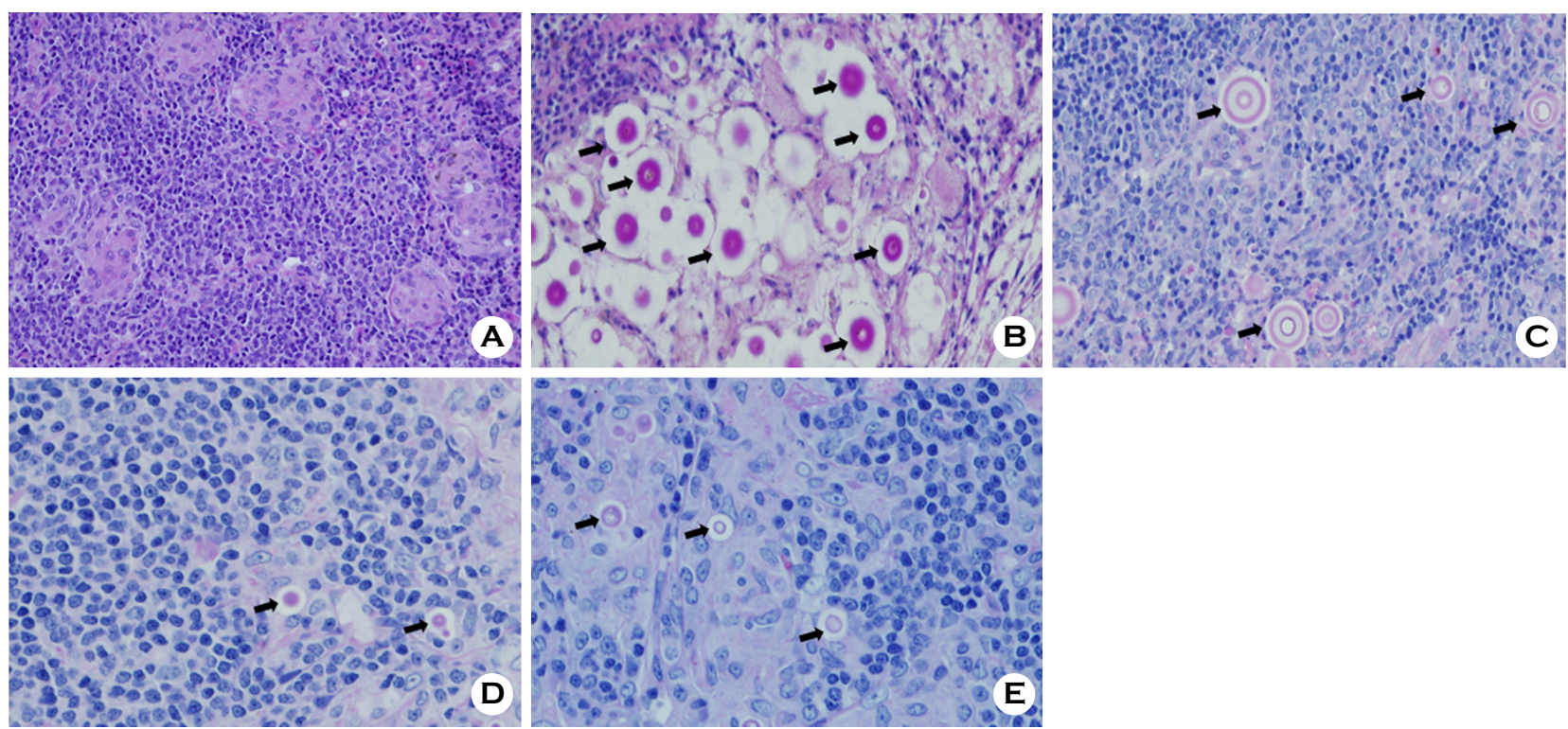

Fig. 6. Spleen sections of rats infected with Cryptococcus species complex showed different histopathological characteristics between experimental groups. (A) Histopathological examination of CS (serotype A, group I) infected rat spleen sections (Periodic acid Schiff stain, $\times 200$ ) (B) Histopathological examination of $C$. gattii (serotype B, group II) infected rat spleen sections (Periodic acid Schiff stain, $\times 200$ ). (C) Histopathological examination of $C$. gattii (serotype C, group III) infected rat spleen sections (Periodic acid Schiff stain, $\times 200$ ). (D) Histopathological examination of $C$. neoformans (serotype D, group IV) infected rat spleen sections (Periodic acid Schiff stain, $\times 200$ ). (E) Histopathological examination of C. neoformans (serotype A/D, group V) infected rat spleen sections (Periodic acid Schiff stain, $\times 200$ ). 

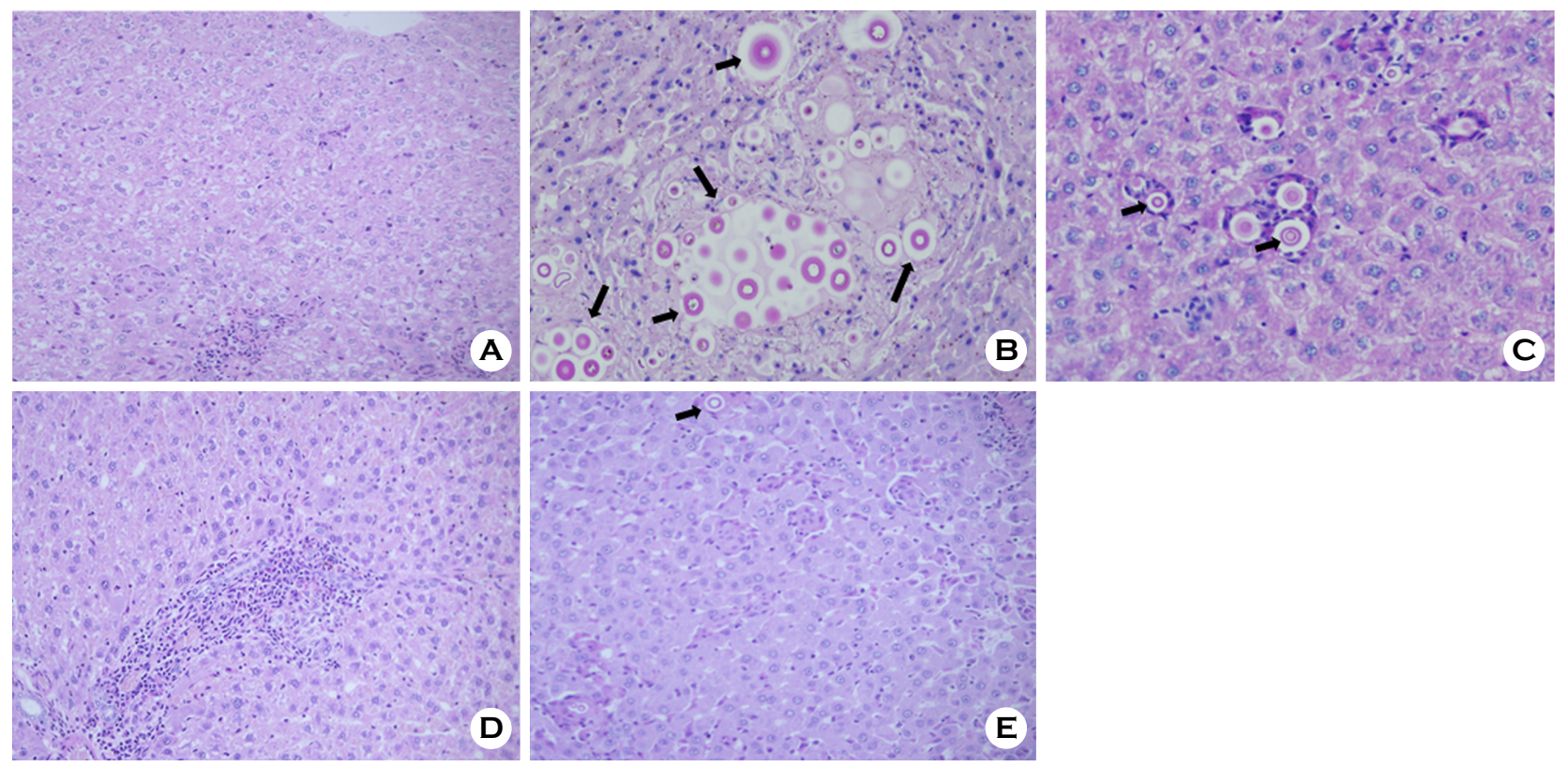

Fig. 7. Liver sections of rats infected with Cryptococcus species complex showed different histopathological characteristics between experimental groups. (A) Histopathological examination of CS (serotype A, group I) infected rat liver sections (Periodic acid Schiff stain, $\times 200$ ). (B) Histopathological examination of $C$. gattii (serotype B, group II) infected rat liver sections (Periodic acid Schiff stain, $\times 200$ ). (C) Histopathological examination of C. gattii (serotype C, group III) infected rat liver sections (Periodic acid Schiff stain, $\times 200$ ). (D) Histopathological examination of $C$. neoformans (serotype D, group IV) infected rat liver sections (Periodic acid Schiff stain, $\times 200$ ). (E) Histopathological examination of C. neoformans (serotype A/D, group V) infected rat liver sections (Periodic acid Schif stain, $\times 200$ ).

Liver lobules that were highly damaged by the infiltration of yeast cells were observed in the liver sections of group II, in addition to macrophages (Fig. 7. (B)). The infiltration of inflammatory cells around the central vein of the liver lobules was observed in group III. In addition, the infiltration of yeast cells was detected around the liver artery and bile duct (Fig. 7. (C)). Yeast cells were not detected in the liver artery and bile duct of the other experimental groups (groups I, IV, and the negative control). However, considerable bleeding, inflammation, and tissue damage were observed in the liver artery and bile duct of groups IV and V, but not in those of group I.

Considerable bleeding and tissue damage were observed in the brain sections of all experimental groups except the control group. Infiltration of yeast cells was detected in groups I and IV, but not in other experimental groups. However, severe brain damage was observed in group II. Slight inflammation and tissue damage were observed in the muscle and testis sections of all groups. However, yeast cells were

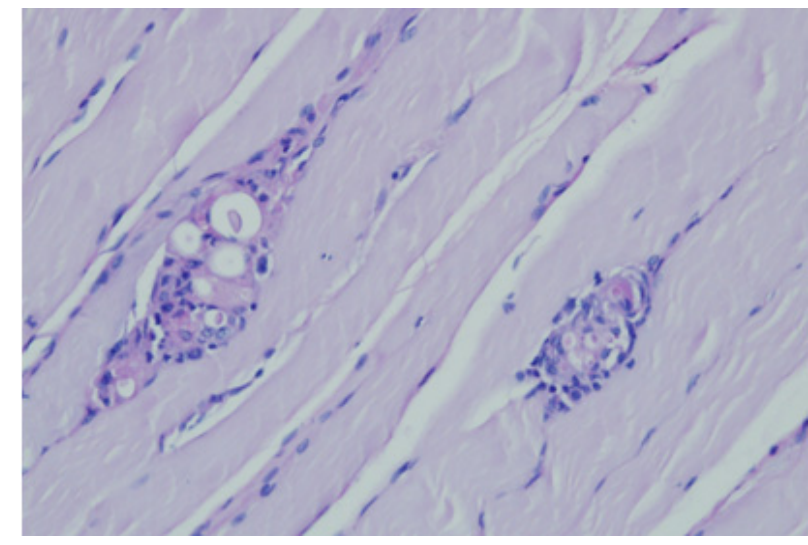

Fig. 8. Histopathological examination of C. neoformans (serotype $\mathrm{A} / \mathrm{D}$ ) infected rat muscle sections (Periodic acid Schiff stain, $X$ 200).

detected only in the muscle sections of group V (Fig. 8). These results suggest that the degree of virulence of the Cryptococcus species complex differs in each organ depending on serotype. 


\section{DISCUSSION}

According to a recent report by the Joint United Nations Program on HIV/AIDS and the World Health Organization, 34 million people have suffered HIV infection, 3 million AIDS patients are diagnosed every year, and 2 million AIDS patients have died from AIDS-related diseases. In particular, the third most frequent complication in AIDS patients is cryptococcal meningitis, where the Cryptococcus species complex is the major etiological agent (Park et al., 2009). The genus Cryptococcus species complex consists of over 30 different species of Cryptococcus. Among these, C. neoformans and $C$. gattii are considered major causative agents of nearly all cryptococcal infections in hosts, but they are not part of the normal flora of human and animal hosts. Other Cryptococcus species have been reported rarely as human and animal pathogens (Hansong and Robin, 2009). In previous research, the etiological agents of cryptococcosis, C. neoformans, and C. gattii species were considered a homogeneous species (Hansong and Robin, 2009; Negroni, 2012). However, these two fungal pathogens have been classified into the five different major serotypes, A, B, C, D and $\mathrm{A} / \mathrm{D}$, based on several differences, including geographical distribution, epidemiology, as well as biochemical and molecular features (Hansong and Robin, 2009; Negroni, 2012).

Many studies reported the occurrence of cryptococcosis caused by C. gattii worldwide (e.g., Asia, Africa, Australia, South and North America, etc.), which previously were not well known as harboring this pathogen. Recently, C. gattii has been considered a crucial pathogen because it mainly infects immunocompetent hosts (Okamoto et al., 2010; Byrnes et al., 2011; Springer et al., 2012; Chen et al., 2013). Furthermore, it was demonstrated that $C$. gattii was more virulent than $C$. neoformans in murine pulmonary cryptococcosis and meningoencephalitis models (Thompson et al., 2012; Okubo et al., 2013).

However, accurate knowledge regarding the differences in the pathogenicity among the five serotypes of Cryptococcus species complex is still limited. In the present study, an intravenous rat model was used to elucidate the different virulence among the five major serotypes of the Cryptococcus species complex. First, we investigated the cumulative mortality of rats infected with five different strains of the Cryptococcus species complex. Previous reports showed that $C$. neoformans strains (serotype $\mathrm{A}, \mathrm{D}$, and $\mathrm{A} / \mathrm{D}$ ) were causative agents of fatal cryptococcal disease. Thompson et al. reported that the median survival in mice infected with C. gattii (10.5 days) was significantly less than in mice infected with C. neoformans (19.5 days). In this study, the cumulative mortality ratio of rats infected with $C$. gatii MYA-4560 (serotype B) was slightly higher than in the other experimental groups. These results suggest that the virulence of $C$. gattii strains might be higher than C. neoformans strains. Although there was no significant difference $(P=$ 0.130 ) in the mortality ratio between rats infected with $C$. gattii (group II and group III) and C. neoformans (group I, group IV, and group V) strains (Fig. 1), mortality by C. gattii should be considered.

Because the five serotype strains in the experimental rats showed different mortalities, the recovery rates of five serotype strains were investigated in the various organs of the rats infected by the Cryptococcus species complex. Cryptococcosis is usually initiated by the inhalation of these fungi, which has a tendency to attack the CNS. Therefore, the lungs and brain are known as major organs of infection by the Cryptococcus species complex (Hansong and Robin, 2009; Negroni, 2012). In this study, the average number of cryptococcal colonies recovered from the lung samples (110.71 $\pm 34.16)$ was significantly higher $(P<0.05)$ than in the other organ samples (Fig. 3). However, the average number of cryptococcal colonies isolated from the brain samples $(59.62 \pm 27.42)$ was not higher than in the other organ samples, including kidney, liver, and spleen. Interestingly, the average number of colonies recovered from the organ samples was different in each group (Table 2). In particular, the average number of colonies recovered in group II was significantly higher than in the other groups in almost all the organ samples except spleen (Fig. 2). In addition, major distributed organs of recovered colonies were specific by each group depending on serotype: lung and liver in groups II and group III; lung and spleen in group III, lung, spleen, and brain in group IV; lung and kidney in group V. to be 
recovered the infected strains as major distributed organs, respectively. Lung was a major recovered organ in group I. The rats infected by serotype B strain showed higher virulence than the rats infected by other serotypes. These results suggest the existence of different infection mechanisms and of differences in the major organs infected by the five serotypes of the Cryptococcus species complex. Further studies are needed to elucidate the reasons for these differences.

The average numbers of the recovered cryptococcal colonies were associated with severe histopathological changes, including bleeding, inflammation, and tissue damage in the organ sections. The degree of histopathological lesions of rats infected with serotype B strain was the most severe, especially in the lung and liver samples. However, the degree of histopathological lesions in rats infected with the serotype A strain was generally slight (Tables 3 and 4). These results suggest that the serotype B strain multiplies rapidly and survives in the lung and liver during infection. The results of the present study indicate that the pathophysiology of the Cryptococcus species complex infection differs according to serotype, and serotype B is the most virulent among the five major serotypes of the Cryptococcus species complex.

In summary, the present study showed that histopathological changes differed in rats infected by five major serotypes of the Cryptococcus species complex. Although there was no significant difference in the mortality ratio between rats infected with the $C$. gattii and $C$. neoformans strains, the mortality, fungal recovery, and histopathological characteristics in the rats infected with serotype B strain were more severe than in the rats infected by other serotype strains. The degree of organ damage (especially in the brain samples) in the group infected with the serotype B strain was more severe than in the other experimental groups. These histopathological characteristics are probably associated with the high virulence of serotype B strain.

\section{ACKNOWLEDGEMENT}

This paper was supported by RESEARCH FUND offered from Catholic University of Pusan. This paper was supported by a research fund granted by the Catholic University of Pusan and by BB21 (Brain Busan 21), Republic of Korea in 2018 .

\section{CONFLICT OF INTEREST}

The authors declare no conflicts of interest.

\section{REFERENCES}

Buchanan KL, Murphy JW. What makes Cryptococcus neoformans a pathogen? Emerg Infect Dis. 1998. 4: 71-83.

Byrnes EJ III, Bartlett KH, Perfect JR, Heitman J. Cryptococcus gattii: an emerging fungal pathogen infecting humans and animals. Microbes Infect. 2012. 13: 895-907.

Chaturvedi V, Chaturvedi S. Cryptococcus gattii: a resurgent fungal pathogen. Trends Microbiol. 2011. 19: 564-571.

Chae HS, Park GN, Kim SH, Jo HJ, Kim JT, Jeoung HY, An DJ, Kim NH, Shin BW, Kang YI, Chang KS. Rapid direct identification of Cryptococcus neoformans from pigeon droppings by nested PCR using CNLAC1 gene. Poult Sci. 2012. 91: 1983 $-1989$.

Chen M, Liao WQ, Wu SX, Yao ZR, Pan WH, Liao Y. Taxonomic analysis of Cryptococcus species complex strain S8012 revealed Cryptococcus gattii with high heterogeneity on the genetics. Clin Med J. 2011. 124: 2051-2056.

Chen M, Pan WH, Boekhout T. Cryptococcus gattii infections in China: extent of the problem? Chin Med J. 2013. 126: 203-205.

Cogliati M. Global Molecular Epidemiology of Cryptococcus neoformans and Cryptococcus gattii: An Atlas of the Molecular Types. Scientifica (Cairo). 2013; 675213.

Hansong M, Robin CM. Virulence in Cryptococcus species (Allen IL, Sima S, Geoffrey MG. Eds). 2009. pp. 131-190. Advances in Applied Microbiology, Academic Press, Burlington.

Hwang SM. Molecular characterization of clinical and environmental strains of Cryptococcus neoformans isolated from Busan, Korea. J Bac Virol. 2010. 40: 91-98.

Lortholary O, Improvisi L, Rayhane N, Gray F, Fitting C, Cavaillon JM, Dromer F. Cytokine profiles of AIDS patients are similar to those of mice with disseminated Cryptococcus neoformans infection. Infect Immun. 1999. 67: 6314-6320.

Negroni R. Cryptococcosis. Clin Dermatol. 2012. 30: 599-609.

Ngamskulrungroj P, Price J, Sorrell T, Perfect JR, Meyer W. Cryptococcus gattii virulence composite: candidate genes revealed by microarray analysis of high and less virulent Vancouver island outbreak strains. PLoS One. 2011. 6: e16076.

Nielsen K, De Obaldia AL, Heitman J. Cryptococcus neoformans mates on pigeon guano: implications for the realized ecological niche and globalization. Eukaryot Cell. 2007. 6: 949-959. 
Okamoto K, Hatakeyama S, Itoyama S, Nukui Y, Yoshino Y, Kitazawa T, Yotsuyanagi H, Ikeda R, Sugita T, Koike K. Cryptococcus gattii genotype VGIIa infection in man, Japan, 2007. Emerg Infect Dis. 2010. 16: 1155-1157.

Okubo Y, Wakayama M, Ohno H, Yamamoto S, Tochigi N, Tanabe K, Kaneko Y, Yamagoe S, Umeyama T, Shinozaki M, Nemoto T, Nakayama H, Sasai D, Ishiwatari T, Shimodaira K, Yamamoto Y, Kamei K, Miyazaki Y, Shibuya K. Histopathological study of murine pulmonary cryptococcosis induced by Cryptococcus gattii and Cryptococcus neoformans. Jpn J Infect Dis. 2013. 66: 216-221.

Park BJ, Wannemuehler KA, Marston BJ, Govender N, Pappas PG, Chiller TM. Estimation of the current global burden of cryptococcal meningitis among persons living with HIV/AIDS. AIDS. 2009. 23: 525-530.
Springer DJ, Phadke S, Billmyre B, Heitman J. Cryptococcus gattii, no longer an accidental pathogen? Curr Fungal Infect Rep. 2012. 6: 245-256.

Thompson GR III, Wiederhold NP, Najvar LK, Bocanegra R, Kirkpatrick WR, Graybill JR, Patterson TF. A murine model of Cryptococcus gattii meningoencephalities. J Antimicrob Chemother. 2012. 67: 1432-1438.

https://doi.org/10.15616/BSL.2018.24.3.183

Cite this article as: Park GN, Kim SY, Kim HR, Jung BK, An DJ, Hong SB, Chang KS. Comparison of Virulence between Five Strains of Cryptococcus Species Complex in a Rat Model. Biomedical Science Letters. 2018. 24: 183-195. 